\title{
Hepatocellular carcinoma-derived exosomal miRNA-21 contributes to tumor progression by converting hepatocyte stellate cells to cancer-associated fibroblasts
}

\author{
Yuan Zhou ${ }^{1+}$, Haozhen Ren ${ }^{1 \dagger}$, Bo Dai ${ }^{1+}$, Jun $\mathrm{Li}^{1}$, Longcheng Shang ${ }^{1}$, Jianfei Huang ${ }^{2^{*}}$ and Xiaolei Shi ${ }^{{ }^{*}}$
}

\begin{abstract}
Background: Hepatocellular carcinoma (HCC) remains a global challenge due to its high morbidity and mortality rates as well as poor response to treatment. The communication between tumor-derived elements and stroma plays a critical role in facilitating cancer progression of HCC. Exosomes are small extracellular vesicles (EVs) that are released from the cells upon fusion of multivesicular bodies with the plasma membrane. There is emerging evidence indicating that exosomes play a central role in cell-to-cell communication. Much attention has been paid to exosomes since they are found to transport bioactive proteins, messenger RNA (mRNAs) and microRNA (miRNAs) that can be transferred in active form to adjacent cells or to distant organs. However, the mechanisms underlying such cancer progression remain largely unexplored.
\end{abstract}

Methods: Exosomes were isolated by differential ultracentrifugation from conditioned medium of HCC cells and identified by electron microscopy and Western blotting analysis. Hepatic stellate cells (HSCs) were treated with different concentrations of exosomes, and the activation of HSCs was analyzed by Western blotting analysis, wound healing, migration assay, Edu assay, CCK-8 assay and flow cytometry. Moreover, the different miRNA levels of exosomes were tested by real-time quantitative PCR (RT-PCR). The angiogenic ability of activated HSCS was analyzed by qRT-PCR, CCK-8 assay and tube formation assay. In addition, the abnormal lipid metabolism of activated HSCs was analyzed by Western blotting analysis and Oil Red staining. Finally, the relationship between serum exosomal miRNA-21 and prognosis of HCC patients was evaluated.

Results: We showed that HCC cells exhibited a great capacity to convert normal HSCs to cancer-associated fibroblasts (CAFs). Moreover, our data revealed that HCC cells secreted exosomal miRNA-21 that directly targeted PTEN, leading to activation of PDK1/AKT signaling in HSCs. Activated CAFs further promoted cancer progression by secreting angiogenic cytokines, including VEGF, MMP2, MMP9, bFGF and TGF- $\beta$. Clinical data indicated that high level of serum exosomal miRNA-21 was correlated with greater activation of CAFs and higher vessel density in HCC patients.

Conclusions: Intercellular crosstalk between tumor cells and HSCs was mediated by tumor-derived exosomes that controlled progression of HCC. Our findings provided potential targets for prevention and treatment of live cancer.

Keywords: Hepatocellular carcinoma, Hepatic stellate cells, Cancer associated fibroblasts, Exosome, miRNA-21, PTEN, AKT, Angiogenesis

\footnotetext{
* Correspondence: jhuang@ntu.edu.cn; njsxl2018@163.com

†Yuan Zhou, Haozhen Ren and Bo Dai contributed equally to this work.

${ }^{2}$ Department of Clinical Biobank, Nantong University Affiliated Hospital, 20,

Xisi Road, Nantong 226001, Jiangsu Province, China

'Department of Hepatobiliary Surgery, Affiliated Drum Tower Hospital of

Nanjing University Medical School, 321, Zhongshan Road, Nanjing 210008,

Jiangsu Province, China
}

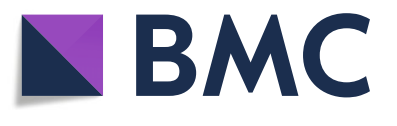

(c) The Author(s). 2018 Open Access This article is distributed under the terms of the Creative Commons Attribution 4.0 International License (http://creativecommons.org/licenses/by/4.0/), which permits unrestricted use, distribution, and reproduction in any medium, provided you give appropriate credit to the original author(s) and the source, provide a link to the Creative Commons license, and indicate if changes were made. The Creative Commons Public Domain Dedication waiver (http://creativecommons.org/publicdomain/zero/1.0/) applies to the data made available in this article, unless otherwise stated. 


\section{Introduction}

Hepatocellular carcinoma (HCC) is responsible for the third most common cancer-related death worldwide [1]. Importantly, the vast majority of $\mathrm{HCC}$ is caused by liver fibrosis or cirrhosis [2]. Liver fibrosis occurs when inactive liver fibroblasts or hepatic stellate cells (HSCs) become activated after liver injury and turn into collagen-producing cells [3]. In HCC, cancer-associated fibroblasts (CAFs) are loosely defined as HSCs found within the tumor mass [4]. These CAFs have been implicated as etiologic players both in the cancer genesis and homeostasis. These data suggest that liver fibrosis, or activated HSCs, plays a crucial role in the development of HCC, which may be similar to the role of CAFs in desmoplastic tumors.

How fibroblasts are activated in HCC remains controversial. Recent studies have revealed multiple potential origins, including activation of stellate cells or portal fibroblasts or transdifferentiation of hepatocytes through epithelial-mesenchymal transition (EMT) [5]. Stemmed from different origins, CAFs are highly heterogeneous, and they can be identified by different specific markers [6]. Among them, $\alpha$-smooth muscle actin ( $\alpha$-SMA) is the most commonly used marker for CAFs [7]. Moreover, CAFs are believed to regulate the inflammatory microenvironment by expressing pro-inflammatory genes, such as IL-1 $\beta$, IL-6, IL- 8 , TGF- $\beta$ and CXCL12 as well as collagen $[8,9]$. The crosstalk between tumor cells and CAFs has been extensively studied [10-12]. However, the mechanisms underlying the activation of HSCs by tumor cells remain largely unexplored in liver cancer.

An important type of cell-cell communication occurs through exosomes. These small, nanometer-sized (50$100 \mathrm{nM}$ ) vesicles of endocytic origin are released into the extracellular milieu by cells under physiological and pathological conditions, including antigen presentation and infectious agent transmission. Tumor exosomes are important mediators of the cross-talk between tumor cells and their microenvironment by sharing genetic information or functional proteins to modulate cellular behavior $[13,14]$. Tumor cell-derived exosomes are involved in the regulation of EMT, tumor angiogenesis, tumor metastasis and radioresistance $[15,16]$.

It has been revealed that microRNA (miRNA) dysregulation greatly contributes to the activation of HSCs [17]. Interestingly, there is a significant overlap in the list of up-regulated or down-regulated miRNAs between HCC tumor tissues and normal tissues [18]. It has been demonstrated that secreted miRNAs can function in a paracrine manner in the surrounding microenvironment, promoting tumor development [19]. Meanwhile, studies have indicated that exosomes contain a high level of miRNAs, and exosomal miRNAs have been shown to contribute to immunomodulation, chemoresistance and metastasis in multiple types of tumor [20, 21]. However, it remains unclear how HSCs are activated through miRNA pathways.

In the present study, we aimed to classify the mechanisms underlying the activation of HSCs in liver cancer. Moreover, CAFs promoted a set of properties of vascular endothelial cells via increasing the secretion of angiogenic factors. The bilateral interaction between primary tumor cells and stromal cells further illuminated a new mechanism of tumor progression and offered new opportunities for potential therapeutic strategies targeting HCC development.

\section{Methods \\ Specimens and primary cells}

Human serum specimens and liver tissues were collected from healthy donors and HCC patients before or after resection in the Nanjing Drum Tower Hospital in Nanjing, China. All procedures were approved by the Ethical Committee of the Nanjing Drum Tower Hospital. Written informed content was obtained from every participant prior to study.

\section{Cell culture}

The human liver cancer cell lines (97H, LM3 and Huh7), the vascular endothelial cell line HUVEC, and the HSC cell line LX2 were purchased from Cell Bank of Type Culture Collection of the Chinese Academy of Sciences (Shanghai Institute of Cell Biology) and maintained in DMEM (Gibco) supplemented with 10\% FBS (Gibco). The normal liver cell line LO2 was purchased from Cell Bank of Type Culture Collection of the Chinese Academy of Sciences and maintained in RPMI-1640 medium (Gibco) supplemented with $10 \%$ FBS (Gibco). All cell lines were cultured at $37^{\circ} \mathrm{C}$ in a humidified incubator containing $5 \% \mathrm{CO}_{2}$. Cell lines were authenticated by short tandem repeats (STR) profiling and confirmed to be mycoplasma negative.

\section{Reagents and antibodies}

Antibodies against CD63 (ab125011, 1:1000), $\alpha$-SMA (ab32575, 1:100), FAP (ab207178, 1:1000), FSP (ab124805, 1:1000), GAPDH (ab8245, 1:10,000), CD9 (ab92726, 1:1000), CD81 (ab79559, 1:1000), FASN (ab22759, 1:500), ATP citrate lyase (EP704Y, 1:1000) and phospho-ATP citrate lyase (T447/S451) (ab53007, 1:1000) and USP2 (ab66556, 1:500) were purchased from Abcam (Cambridge, MA, USA). Antibodies against phosphorPTEN (Ser380/Thr382/383) (9554S, 1:1000), total PTEN (7960 T, 1:1000), PDK1 (3062 T, 1:1000), phospho-PDK1 (Ser241) (3438 T, 1:1000), phospho-Akt (Ser473) (4060 T, 1:1000) and AKT (4691 T, 1:1000) were supplied by Cell Signaling Technology (Beverly, MA, USA). AKT inhibitor MK-2206 was provided by Selleck (Houston,USA). 


\section{Western blotting analysis}

Whole-cell protein extracts were homogenized in lysis buffer and centrifuged at 12,000 r.p.m. for 15 min. Protein concentrations were determined by bicinchoninic acid (BCA) assay. After immunoblotting, the proteins were transferred onto nitrocellulose membranes, followed by incubation with specific antibodies. The immunocomplexes were then incubated with the fluorescein-conjugated secondary antibody, and immunoreactive bands were visualized by an Odyssey fluorescence scanner (Li-Cor, Lincoln, NE).

\section{RNA interference}

siRNAs and mimics of indicated miRNAs were obtained by RiboBio Company (Guanngzhou, China). The sequences of siRNAs and miRNA mimics referred above were listed in Additional file 1: Table S1. Transfection with siRNAs and miRNAs was completed using riboFECT $^{\mathrm{m}} \mathrm{CP}$ (RiboBio) according to the manufacturer's instructions.

\section{Animal studies}

To examine the roles of exosomes in CAFs, $1 \times 10^{6}$ Huh7 cells were intravenously injected into male nude mice through the tail vein (Chinese Science Academy, Shanghai, China). Subsequently, mice were randomly divided into groups and intravenously injected with equal numbers of exosomes from different tumor cells twice a week for 1 month. For xenograft assays, $1 \times 10^{6}$ Huh7 cells and $1 \times 10^{6}$ LX2 cells were injected subcutaneously into the right side of each male nude mouse (Chinese Science Academy). The sizes of tumors (length $\times$ width: $2 \times 0.5$ ) were measured at the indicated time points, and tumors were obtained at 4 weeks after injection. All animal experiments were approved by the University Committee on Use and Care of Animals of Nanjing Drum Tower Hospital.

\section{Cell viability assay, migration assay and wound-healing assay}

For cell viability assay, Cell Counting Kit 8 (CCK-8) assay (Dojindo Laboratories, Kumamoto, Japan) was used to assess cell viability according to manufacturer's instructions. For migration assay, $5 \times 10^{4} \mathrm{LX} 2$ cells were plated into 24-well transwell plates with inserts (Corning). The medium in inserts was free of FBS, whereas the medium outside the inserts was supplemented with $10 \%$ FBS. To detect exosome function, equal quantities of tumor-derived exosomes were added into the inserts. After $24 \mathrm{~h}$, the cell inserts were fixed and stained according to manufacturer's protocols. Representative fields were photographed, and the number of migrated cells per field was counted. For wound-healing assay, equal numbers of LX2 cells were plated into six-well plates. Then the cell monolayers were wounded with a pipette tip to draw a gap on the plates. After treated with tumor-derived exosomes, fibroblasts that migrated into the cleared section were observed under microscope at the specific time points.

\section{RNA extraction and qRT-PCR}

HSC RNA was extracted from snap-frozen liver tissues with TRIzol ${ }^{\text {Tw }}$ reagent (Life Technologies, USA) according to the manufacturer's instructions. Briefly, cells in six-well plate were homogenized in $1 \mathrm{~mL}$ TRIzol $^{\mathrm{m}}$ reagent at room temperature. Then $200 \mu \mathrm{L}$ trichloromethane was added and mixed thoroughly, followed by centrifugation at $12,000 \times \mathrm{g}$ for $15 \mathrm{~min}$ at $4{ }^{\circ} \mathrm{C}$. The upper clear phase was collected and added with $500 \mu \mathrm{L}$ isopropanol, followed by centrifugation at $12,000 \times \mathrm{g}$ for $10 \mathrm{~min}$ at $4{ }^{\circ} \mathrm{C}$. The liquid supernatant was discarded, and the pellet was washed with $75 \%$ ethanol. The RNA was re-suspended in $50 \mu \mathrm{L}$ RNase-free DEPC-water and stored at $-80^{\circ} \mathrm{C}$.

Reverse transcription was performed with PrimeScript $^{\text {tw }}$ RT Master Mix (Takara, Japan) according to the manufacturer's instructions. qRT-PCR was performed using TB Green ${ }^{\text {TM }}$ Premix Ex Taq ${ }^{\text {Tw }}$ (Takara, Japan) on an ABI PRISM 7500 real-time PCR System (Applied Biosystems, USA). Primers used for $\mathrm{qPCR}$ are shown in Additional file 1: Table S1. The relative expression levels of mRNAs were calculated with $2^{-\Delta \Delta C t}$ method. GAPDH was selected as the housekeeping gene.

miRNeasy Mini Kit (Qiagen) was used to extract RNA from exosomes in plasma/medium according to the manufacturer's instructions, and cel-miR-39 (Takara) was added into each sample at a final concentration of $10 \mathrm{pmol} / \mu \mathrm{L}$ acting as external reference. Total RNA was stored at $-80^{\circ} \mathrm{C}$ for subsequent experiments.

Reverse transcription and qRT-PCR for exosomal miRNA, as well as internal reference U6 were performed using miRNA RT-PCR Quantitation Kit (Qiagen) according the manufacturer's instructions. Briefly, after an initial denaturation step at $95^{\circ} \mathrm{C}$ for $3 \mathrm{~min}$, the amplifications were carried out with 40 cycles at a melting temperature of $95^{\circ} \mathrm{C}$ for $15 \mathrm{~s}$, and an annealing temperature of $62^{\circ} \mathrm{C}$ for $34 \mathrm{~s}$. The relative expression levels of exosomal miRNAs were calculated with $2^{-\Delta \Delta C t}$ method.

\section{Oil red staining}

Cells were fixed in $10 \%$ formalin, washed with $60 \%$ propylene glycerol, and then stained with $0.5 \%$ oil red $\mathrm{O}$ (Sangon Bio) in propylene glycerol for $10 \mathrm{~min}$ at $60^{\circ} \mathrm{C}$. The red lipid droplets were visualized by microscopy.

\section{Immunohistochemistry and in situ hybridization analysis}

For immunohistochemistry, the slides were incubated with above-mentioned primary antibodies, followed by incubation with horseradish peroxidase-conjugated secondary 
antibodies (Santa Cruz Biotechnology). Finally, the staining processes were performed with diaminobenzidine colorimetric reagent solution (Dako, Carpinteria, USA) and hematoxylin (Sigma Chemical Co., USA). For in situ hybridization analysis, hsa-miRNA-21 miRCURY LNA detection probe (Exiqon, Denmark) was used, and the total staining processes were carried out according to manufacturer's protocols. Images were captured with Aperio ScanScope AT Turbo (Aperio, USA) and assessed with image-scop software (Media Cybernetics, Inc.).

\section{Immunofluorescence analysis}

Immunofluorescence analysis was performed according to previously established protocols. Cells were seeded into 24-well dishes and fixed by $4 \%$ paraformaldehyde $24 \mathrm{~h}$ later. Fixed cells were stained with $\alpha$-SMA and FAP antibodies (mentioned above), followed by incubation with FITC-conjugated anti-mouse IgG and Cy3-conjugated anti-rabbit IgG (Abcam). Representative images were detected by fluorescence microscopy (Leica, German), and data were processed via Photoshop.

\section{Tube formation assay}

EC matrix was thawed at $4{ }^{\circ} \mathrm{C}$ overnight, and required wells of pre-chilled 96-well plates were coated with $50 \mu \mathrm{L}$ diluted EC matrix and incubated at $37^{\circ} \mathrm{C}$ for $1 \mathrm{~h}$ to solidify. Subsequently, $150 \mu \mathrm{L}$ of HUVEC cells $\left(1 \times 10^{4}\right)$ with different conditional media were added to the solidified matrix and incubated at $37{ }^{\circ} \mathrm{C}$ for $12 \mathrm{~h}$. Endothelial cell formation was observed using a microscope. Focus was placed on distinct areas, and the tubes formed were counted according to the kit procedure.

\section{Isolation and analysis of exosomes}

For exosome isolation, equal numbers of different cells were transplanted into $10-\mathrm{cm}$ plates and maintained in fresh DMEM supplemented with serum, which was depleted of exosomes by centrifugation at $12,000 \times \mathrm{g}$ overnight. After $48 \mathrm{~h}, \mathrm{CM}$ was collected and filtrated through $0.22-\mu \mathrm{m}$ filters (Millipore, USA). Exosomes in CM or serum samples were isolated by ultracentrifugation according to the standard methods described previously [22]. Ultracentrifugation experiments were performed with Optima MAX-XP (Beckman Coulter, USA). Exosomes were observed by Philips CM120 BioTwin transmission electron microscope (FEI Company, USA).

\section{Exosomes tracing}

For exosome-tracing experiments, tumor cells were pre-treated by PKD67 (Beyotime, China), and exosomes in $\mathrm{CM}$ were obtained as described above. After incubation with recipient cells that were pre-treated with FAP antibody and DAPI (Beyotime), exosomes were observed by fluorescence microscope (Leica, German).

\section{Transmission electron microscopy}

The obtained exosomes were fixed with fixative buffer containing $2 \%$ paraformaldehyde and $2.5 \%$ glutaraldehyde in $0.1 \mathrm{M}$ PBS. After embedding, samples were cut into $0.12-\mu \mathrm{m}$ sections and stained with $0.2 \%$ lead citrate and $1 \%$ uranyl acetate. The images were detected by a JEOL TEM-2000 EX II (JEOL, Tokyo, Japan).

\section{Cell cycle analysis}

HSCs were seeded into six-well plates at a density of $1.5 \times 10^{5}$ cells/well overnight, and the cells were conditionally cultured for $24 \mathrm{~h}$. Cell cycle was detected using cell cycle analysis kit (Thermo Fisher Scientific, USA) according to the manufacture's protocols and measured by flow cytometry.

\section{Collagen contraction assay}

A total of $2 \times 10^{5}$ HSCs were suspended in $100 \mu \mathrm{L}$ DMEM. Then the cell suspension was mixed with $100 \mu \mathrm{L}$ of collagen mix containing $68.75 \mu \mathrm{L}$ DMEM, $0.72 \mu \mathrm{L} 1 \mathrm{~N} \mathrm{NaOH}$ and $31.25 \mu \mathrm{L}$ type 1 rat tail collagen (Corning), added to one well of 6-well plates and allowed to solidify for $45 \mathrm{~min}$ at $37^{\circ} \mathrm{C}$. After incubation with medium containing $\mathrm{HCC}$-derived exosomes, the gels were photographed by digital camera.

\section{Statistical analysis}

Data analysis was performed using the SPSS software version 16. Each experiment was carried out in triplicate at least, and all results were presented as mean \pm SD. $x^{2}$-Test and Student's t-test were used to assess statistical significance. Kaplan-Meier analysis and log-rank tests were applied for survival analysis. A $p$ value of $<0.05$ was considered as statistically significant.

\section{Results}

Tumor-derived exosomes regulate activation of HSCs

CAFs have been demonstrated to actively participate in the tumor invasion. Based on the high expression of $\alpha$-SMA, the most effective marker, similar results were observed in tissues from HCC patients (Fig. 1a) and in orthotopically implanted tumors in mice (Fig. 1b). Compared with the high expression of $\alpha$-SMA in HCC tissue, the expression of $\alpha$-SMA was negative in normal tissue (Additional file 2: Figure S1a). Therefore, it was very important to further understand the activation of CAFs caused by tumor cells. Tumors mainly induce systemic changes through exosome secretion [23]. However, whether exosomes participate in the activation of HSCs remains largely unexplored. In the present study, two liver cancer cell lines (97H and LM3) and one liver cell line (LO2) were employed to clarify this question. First, we purified exosomes from tumor cell-conditioned medium through ultracentrifugation, a standard exosome isolation 
a a-SMA in human HCC tumor

Patient 1
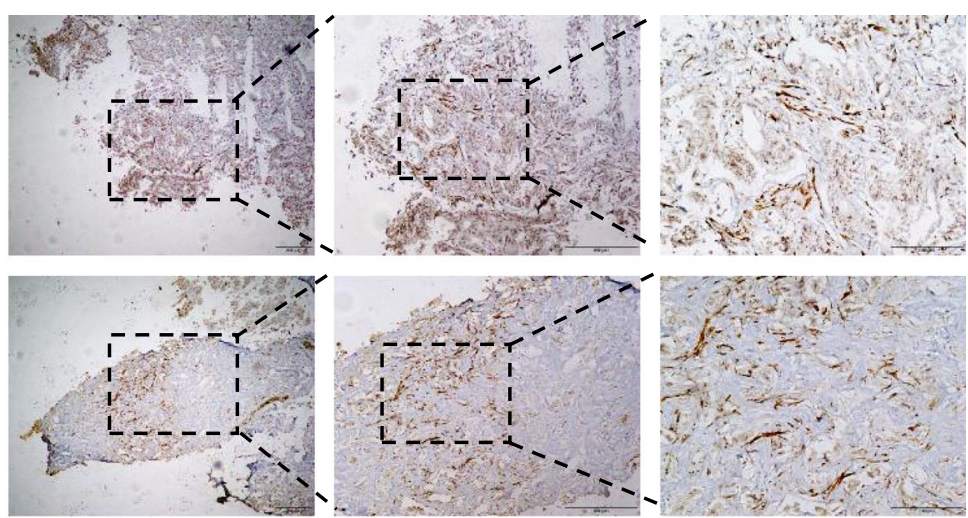

b

a-SMA in mice HCC xenograft tumor

LM3
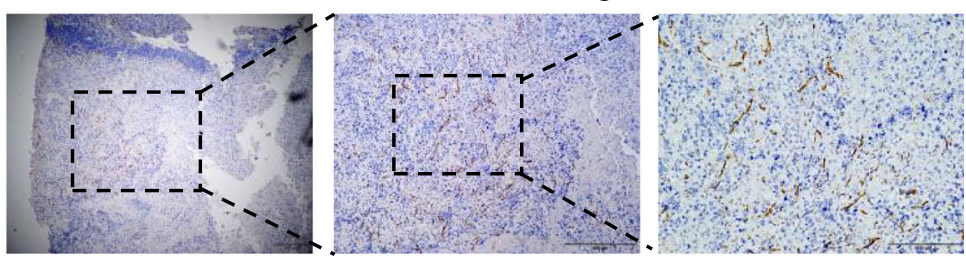

$97 \mathrm{H}$
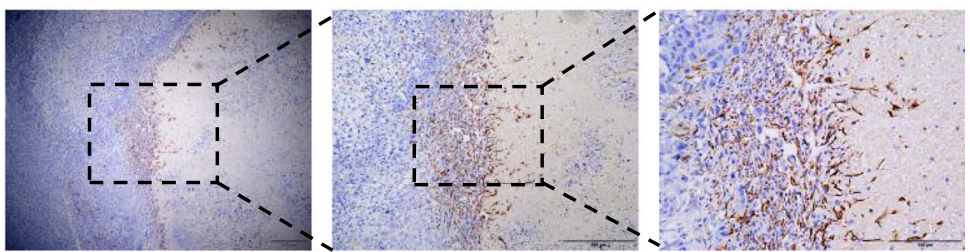

C
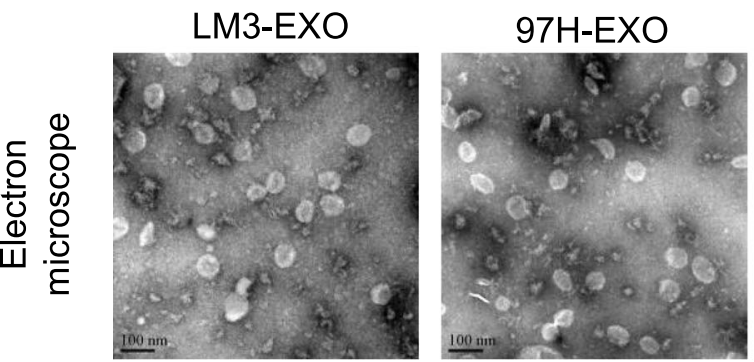

LO2-EXO

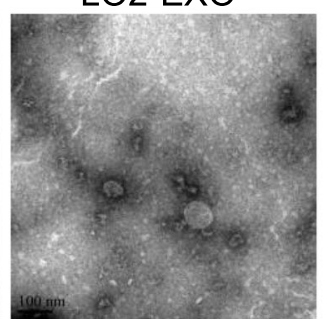

d
\begin{tabular}{|c|c|}
\hline Cell & $\begin{array}{c}\text { Exosome diameter } \\
(\mathrm{nm})\end{array}$ \\
\hline LM3 & $70.6 \pm 8.9$ \\
\hline $97 \mathrm{H}$ & $75.3 \pm 10.5$ \\
\hline LO2 & $78.4 \pm 3.6$ \\
\hline
\end{tabular}

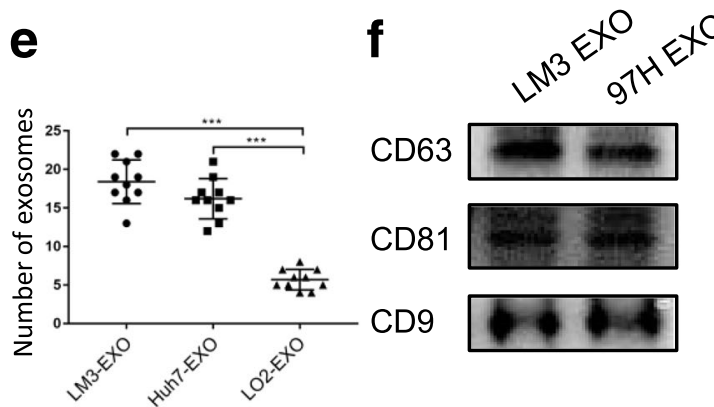

Fig. 1 Tumor-derived exosomes activated HSCS. a, b Representative images of a-SMA staining in primary HCC and in orthotopically implanted tumors in mice. c, $\mathbf{d}$, e Exosomes released by different cells were detected by electron microscopy. $\mathbf{f}$ Immunoblotting assay of indicated proteins in exosomes from different cancer cells. Each experiment was performed three times independently, and results are presented as mean \pm s.d. Student's t-test was used to analyze the data. $\left({ }^{*} p<0.05 ;{ }^{* *} p<0.01 ;{ }^{* * *} p<0.001\right)$ 
method. The cup-shaped structure, size and number of isolated exosomes were identified by electron microscopy (Fig. 1d-f). Intriguingly, we demonstrated that much more exosomes were secreted from HCC cells compared with LO2 cells (Fig. 1e). In addition, the detection of characteristic CD63, CD81 and CD9 further verified that the isolated particles were exosomes (Fig. 1f).

As a human cell line of HSCs, LX2 was selected as normal HSCs in our study. To identify the delivery of exosomes, we labeled the tumor-derived exosomes with PKH67 (green). After incubation, immunofluorescence imaging showed the presence of green spots in recipient HSCs, suggesting that labeled exosomes released by different cells were delivered to HSCs (Fig. 2a). Meanwhile, the expression of $\alpha$-SMA was high, suggesting that HSCs were activated by tumor cell-derived exosomes, not by liver cell-derived exosomes (Fig. 2a). Western blotting analysis revealed that HSCs were activated by HCC cell-derived exosomes in a concentration-dependent manner (Fig. 2b and c). Moreover, we showed that HCC cell-derived exosomes were positively correlated with the activation of HSCs. Since the increased cell proliferation is a manifestation of activated HSCs, we examined whether HCC cell-derived exosomes affected the proliferation of HSCs. CCK-8 assay indicated that HCC cell-derived exosomes significantly promoted the cell growth in a time- dependent manner (Fig. 2d). To further evaluate the different abilities of educating HSCs among the exosomes derived from different cells, Edu incorporation assays and flow cytometry assays showed that the proportion of HSCs in the S phase was increased, suggesting that the cell proliferation was increased under the stimulation of HCC cell-derived exosomes (Fig. 2e-g and Additional file 3: Figure S2a). Wound-healing assay further confirmed that HCC cell-derived exosomes could remarkably improve the migration ability of HSCs (Fig. 3a). Migration assay showed that more HSCs migrated in the $97 \mathrm{H}-\mathrm{EXO}$ group and LM3-EXO group compared with the control group and LO2 group, respectively (Fig. 3b). The contraction abilities of HSCs were markedly enhanced after treatment with HCC cell-derived exosomes compared with LO2 cell-derived exosomes or control (Additional file 3: Figure S2b). Moreover, high expression levels of proimflammatary factors were found in the activated HSCs (Additional file 3: Figure S2c). In addition, intravenous exosomes from LM3 or $97 \mathrm{H}$ markedly contributed to the growth of subcutaneous implanted tumor (mixed injection of HCC-Huh7 and LX2) in nude mice, with more nodules detected at the same time (Fig. 3c-h). Immunofluorescence assay indicated that PKH67-labeled exosomes (green) existed in CAFs which expressed FAP (red) (Additional file 4: Figure S3a). Ki67 staining showed that the proliferation of CAFs was increased under the stimulation of HCC exosomes (Additional file 5: Figure S4a). Taken together, the above-mentioned results suggested that exosomes derived from highly metastatic HCC cells greatly contributed to the activation of HSCs to foster CAFs.

\section{MiRNA-21 in exosomes mediates activation of HSCs}

We next explored how tumor-derived exosomes activated HSCs. MiRNAs encapsulated in exosomes are abundant, playing an important role in cell-cell communication [24]. Therefore, we hypothesized that tumor-derived exosomal miRNAs mediated the activation of HSCs. Five types of miRNAs, which are closely related to liver cancer [25], were selected to verify this hypothesis. Quantitative reverse-transcriptase PCR (qRT-PCR) analysis further confirmed the elevated expression of miRNA-21 in both cancer cell-derived exosomes (Fig. 4a) and HCC cells (Additional file 6: Figure S5a). When the HCC cell-derived exosomes were added into the culture medium of LX2, the expression of miRNA-21 in HSCs was increased, coupled with the activation of HSCs (Additional file 7: Figure S6a). Furthermore, miRNA-21 mimic also contributed to potential activation of HSCs (Fig. 4b-f, Additional file 7: Figure S6b-e). To further investigate the role of miRNA-21, HCC cells were transfected with miRNA-21 inhibitor. After the transfection, the expression of miRNA-21 in HCC cell-derived exosomes was reduced (Fig. 4g). As expected, the effect of miRNA-21 on HSC activation was abolished by siRNA-miRNA-21 (Fig. 4h, Additional file 8: Figure S7 and Additional file 9: Figure S8). Collectively, these findings revealed that tumor-derived exosomal miRNA-21 mediated the activation of HSCs.

\section{Exo-MiRNA-21 activates HSCs via PTEN/PDK1/AKT axis and lipogenesis}

Previous experiments have shown that PTEN (gene of phosphate and tension homology deleted on chromsome ten), a well known tumor-suppressor gene, is the target for miRNA-21 [26]. Therefore, we assessed the PTEN expression in our experimental models. Figure $5 \mathrm{a}$ and $\mathrm{b}$ exhibit that exosomes of HCC cells reduced phosphorylated level of PTEN, along with the increased expression of its downstream inhibitory protein PDK1 (3-phosphoinositide-dependent protein kinase-1) compared with the control group. Furthermore, miRNA-21 suppression also promoted the PTEN phosphorylation and reduced the PDK1 phosphorylation (Fig. $5 \mathrm{c}$ and d). Meanwhile, PTEN is a negative regulator of v-akt murine thymoma viral oncogene homo$\log$ (Akt) pathway [26]. Akt pathway is believed to be involved in the regulation of HCC [27]. To examine whether AKT pathway was regulated by miRNA-21 in activated HSCs, we examined the phosphorylation of Akt. Figure 5a and $b$ show that the phosphorylation of AKT was increased 


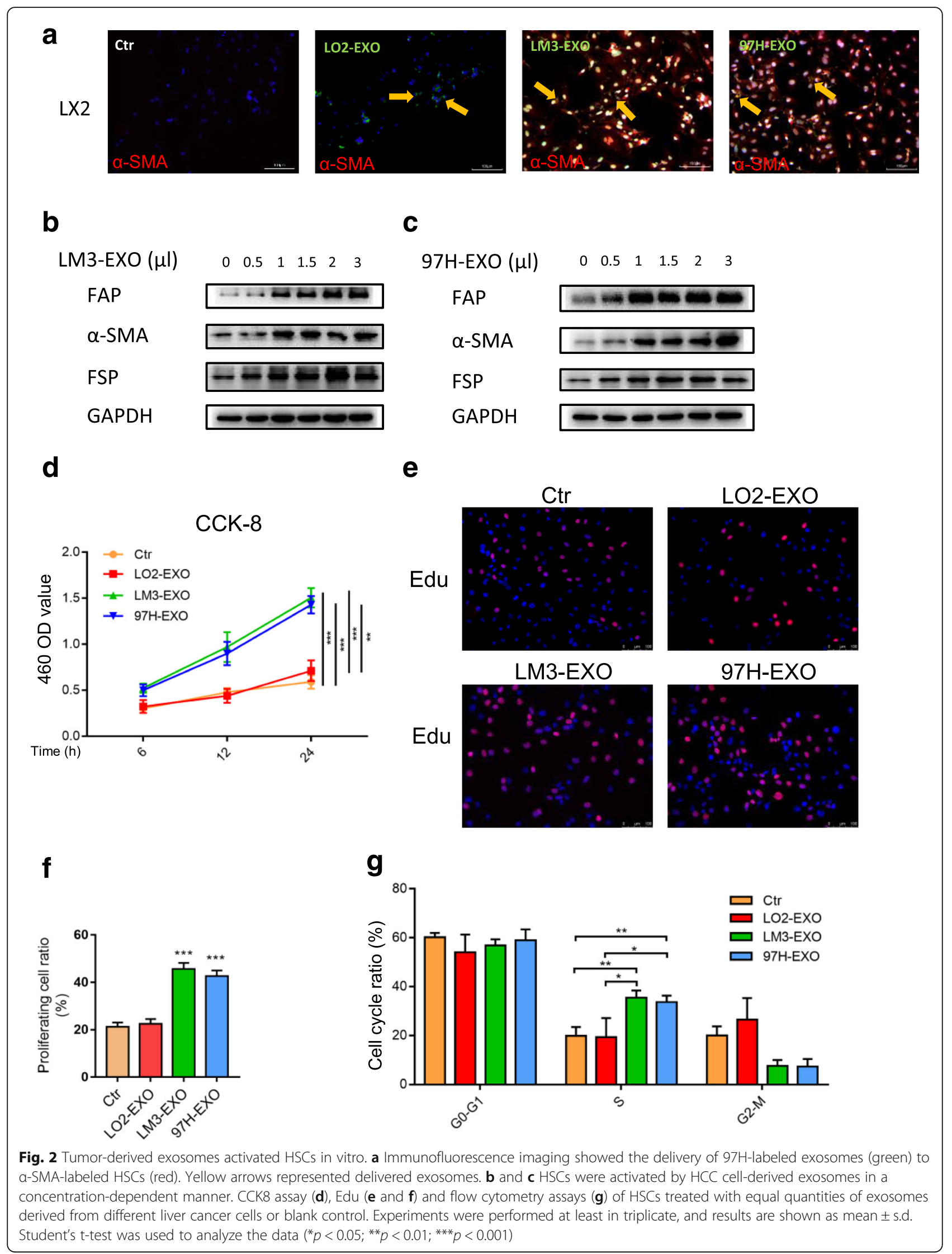




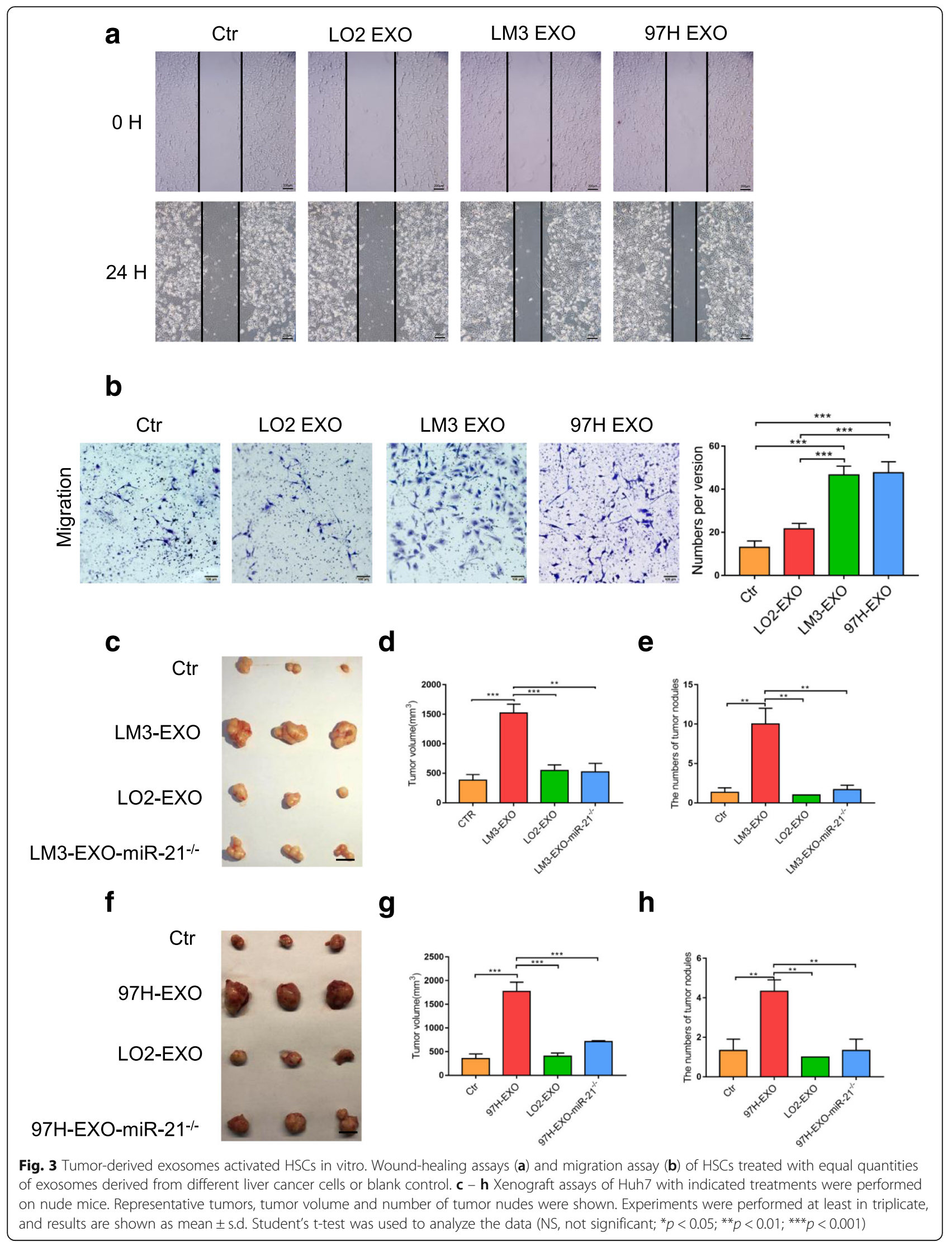




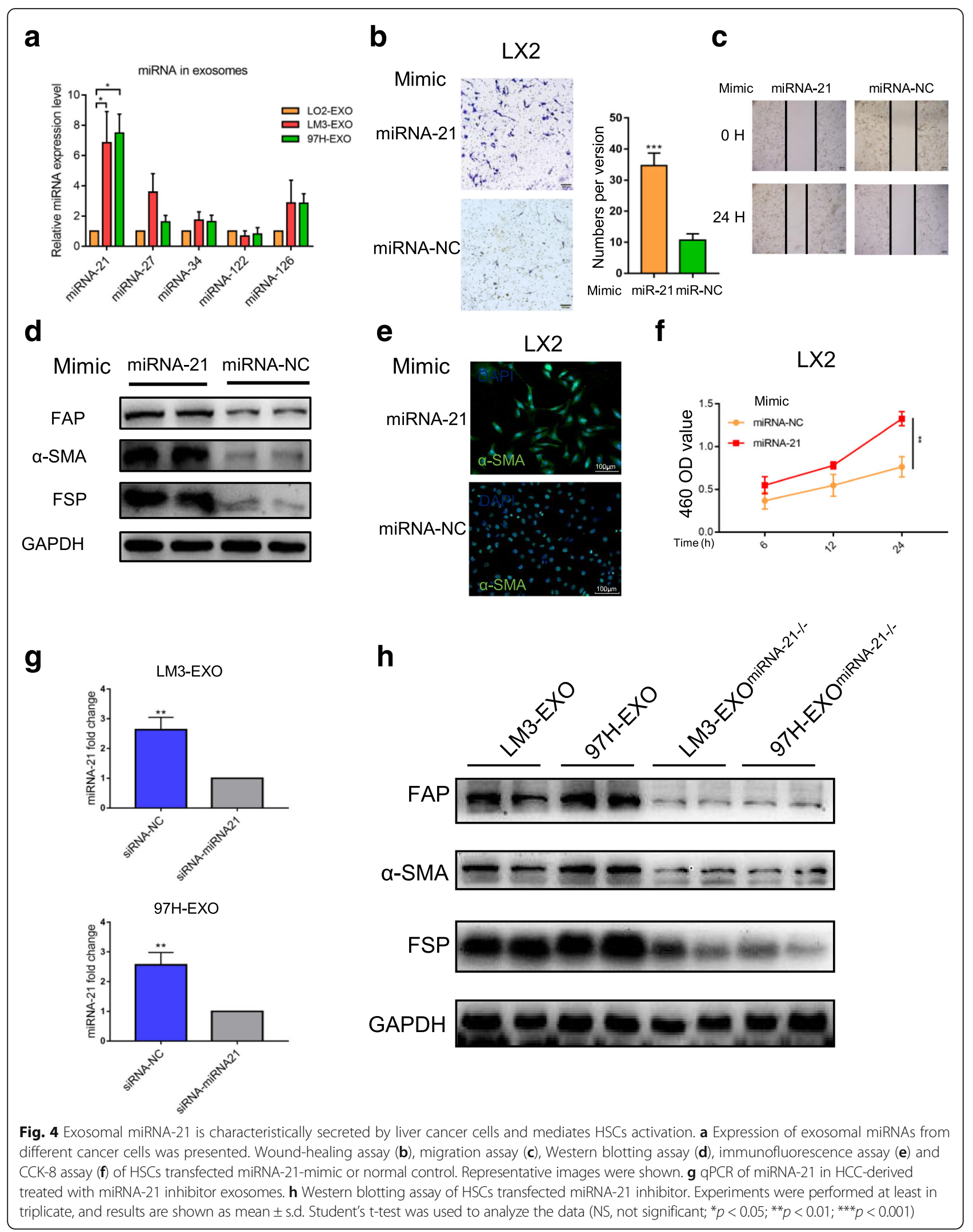




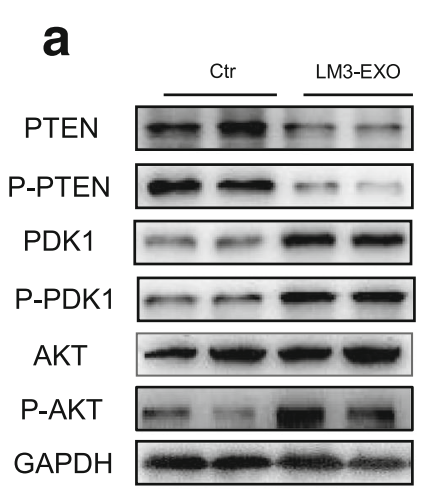

b
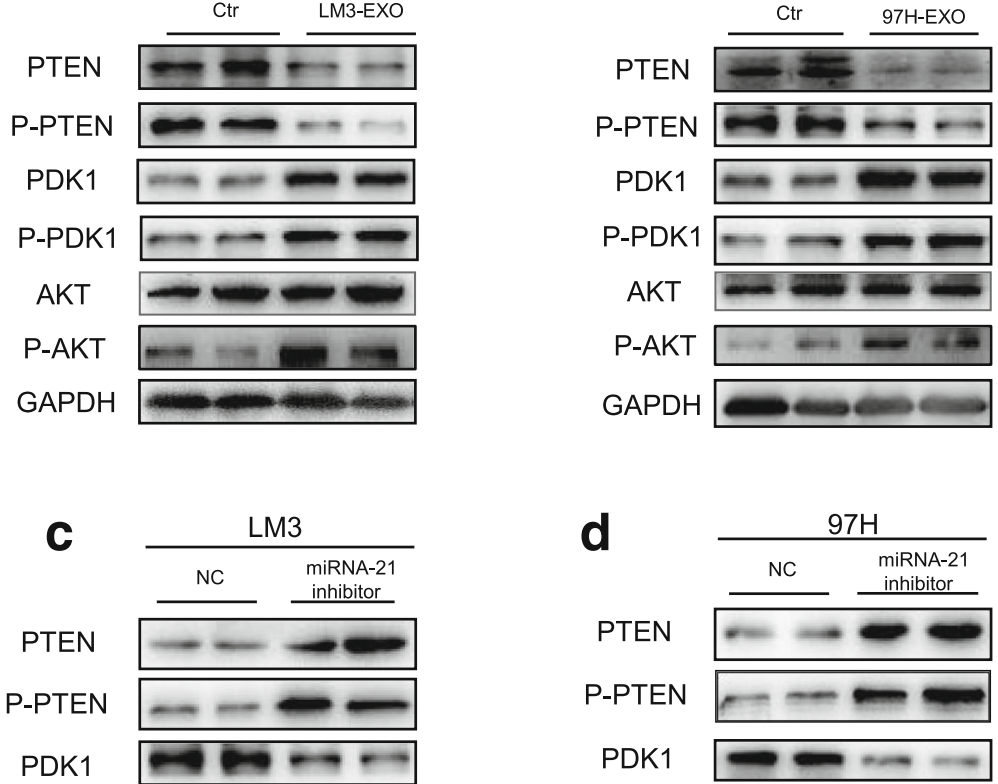

P-PDK1

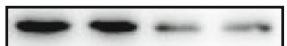

AKT $\longrightarrow \sim$

P-AKT $\longrightarrow$

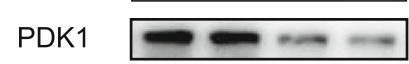

GAPDH

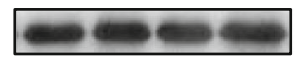

e

P-PDK1

AKT

P-AKT

GAPDH
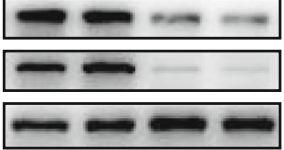

LM3-EXO 97H-EXO LM3-EXO 97H-EXO AKT inhibitor AKT inhibitor

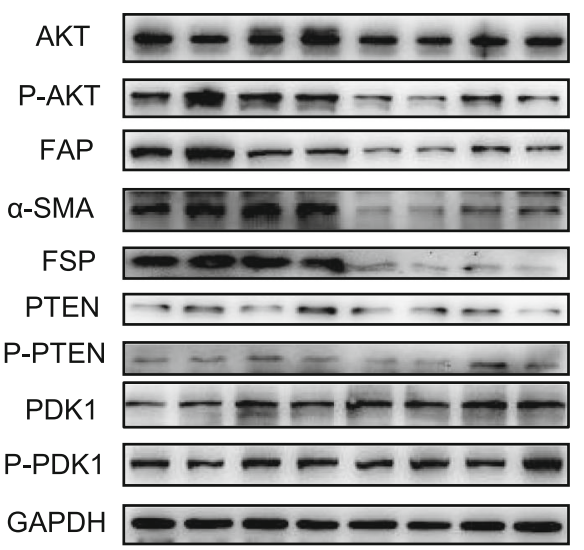

Fig. 5 Exosomal miRNA-21 activates HSCs via PTEN/PDK1/AKT signaling axis. a, b Immunoblotting assays of indicated proteins in HSCs treated with control or exosomes from different tumor cells. c-e Western blotting assays of indicated proteins in HSCs with indicated treatments. Each experiment was performed in triplicate, and data are presented as mean \pm s.d. Student's t-test was used to analyze the data $\left({ }^{*} p<0.05\right.$; $\left.{ }^{* *} p<0.01 ;{ }^{* *} p<0.001\right)$

in HCC-exosome-miRNA-21-activated HSCs. The abovementioned results indicated that PTEN/PDK1/Akt signaling might play a role in miRNA-21-induced stimulation in HSCs. When the MK-2206, an efficient inhibitor of AKT, was added to the culture medium of HSCs, the activation of HSCs caused by cancer cell-derived exosomes was reversed (Fig. 5e and Additional file 8: Figure S7 and
Additional file 9: Figure S8). These results confirmed that HCC-exosome-miRNA-21 regulated the activation of HSCs through PTEN/PDK1/Akt pathway.

Aberrant lipogenesis has been linked to metabolic abnormalities, such as diabetes, obesity and the metabolic syndrome as well as cancer [28]. In the latter disease, unconstrained lipogenesis is necessary to 
maintain a constant supply of lipids and lipid precursors to fuel membrane production and lipid-based post-translational modification of proteins in a context of elevated proliferation. At the molecular level, exacerbated lipogenesis is reflected by the coordinately increased activity and expression of lipogenic enzymes in neoplastic cells, such as ATP citrate lyase (ACLY) and fatty acid synthase (FASN). Previous study has demonstrated that activated AKT also regulates the enzymatic activities of ACLY and FASN [29]. Therefore, we investigated the levels of lipogenic pathway enzymes, ACLY and FASN, by immunoblotting. We found that a progressive induction of FASN and ACLY occurred in tumor and para-tumor (Fig. 6a). Previously, it has been shown that ubiquitin-specific protease 2a (USP2a) sustains FASN activity by impeding its ubiquitin-dependent degradation [30]. Therefore, we determined the USP2a level in our samples. Our data showed that the USP2a level was up-regulated in tumor and para-tumor as well (Fig. 6a). Considering that the CAFs are mostly localized on the periphery of the tumor, we then assessed the activation of HCC cell-derived exosomes in HSCs by immunoblotting. Compared with the control group, cancer cell-derived exosomes were significantly and positively correlated with lipogenesis since the levels of ACLY, FASN and USP2a were increased in exosome-challenged HSCs (Fig. 6b). Moreover, the activation of HSCs was abolished by miRNA-21 inhibitor and AKT inhibitor (Fig. 6b). To further examine whether cancer cell-derived exosomes were associated with lipogenesis in HSCs, we assessed the severity of steatosis by oil eed assay. Compared with control group and LO2-EXO group, we observed that LM3-EXO and 97H-EXO led to increased levels of lipid staining in HSCs, whereas the staining was significantly lower in miRNA-21 inhibitor group and AKT inhibitor group (Fig. 6c). Collectively, the present data indicated that there was a positive correlation between cancer cell-derived exosomes and lipid contents in HSCs.

\section{Activated HSCs promote angiogenesis}

The transition from pre-angiogenic to macroscopic metastasis is a rate-limiting step in the hepatic metastatic cascade [31], which can be used to identify the development of an angiogenic phenotype as a key factor for metastatic progression within the liver. CAFs can promote cancer progression through various mechanisms, including the stimulation of angiogenesis. Under certain

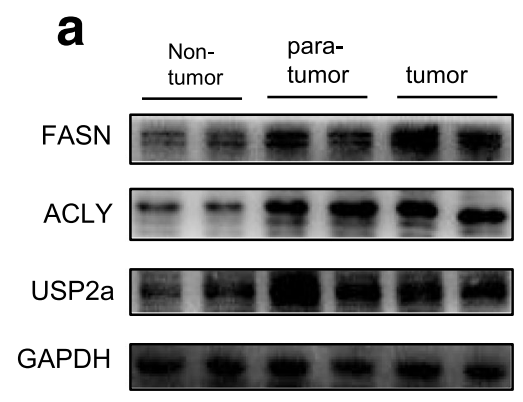

C
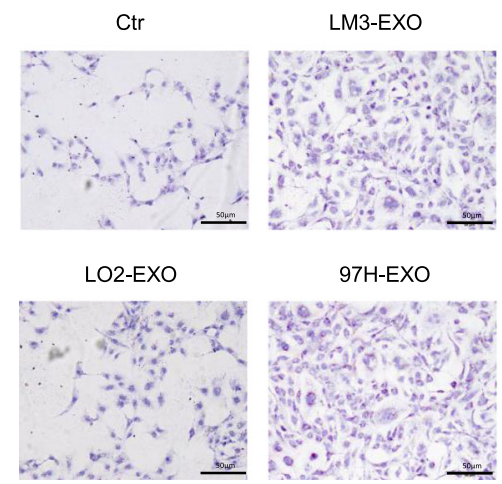

$97 \mathrm{H}-\mathrm{EXO}$

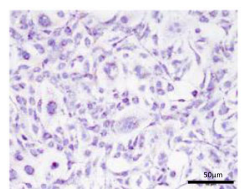

b
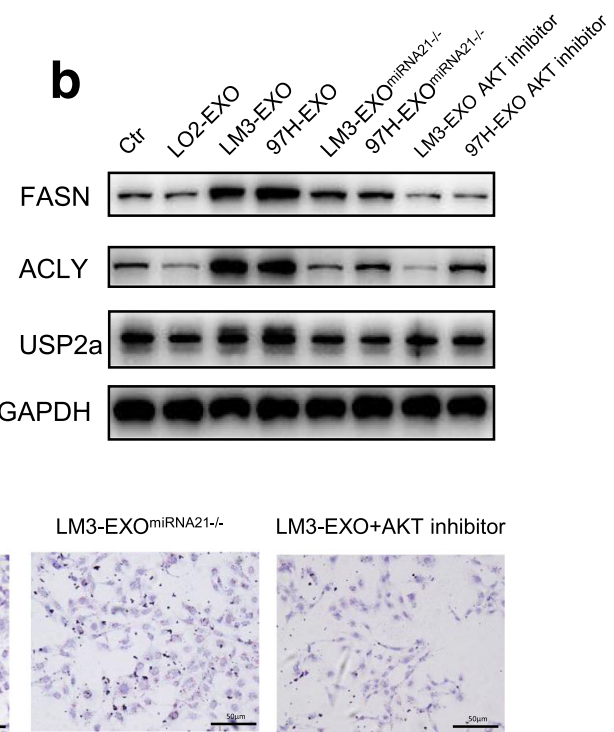

97H-EXOMiRNA21-

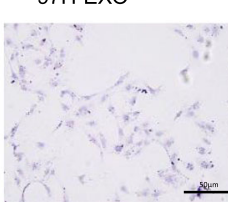

LM3-EXO+AKT inhibitor

97H-EXO+AKT inhibitor

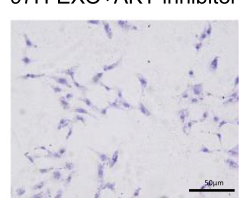

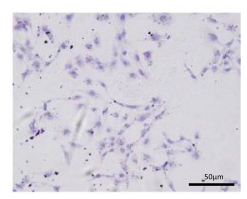

Fig. 6 HCC derived exosomes induced abnormal lipid metabolism. a, b Western blotting assays of lipid metabolism related proteins in HCC patients or HSCs with different stimulations. c Oil Red staining assay showed the abnormal lipid accumulation in HSCs with indicated treatments. Each experiment was performed in triplicate, and data are presented as mean \pm s.d. Student's t-test was used to analyze the data $\left(^{*} p<0.05\right.$; $\left.{ }^{* *} p<0.01 ;{ }^{* *} p<0.001\right)$ 
circumstances, CAFs promote tumor angiogenesis. However, they have not been linked to angiogenesis, especially in the hepatic metastatic microenvironment. We assessed the vasculature in HCC mice using immunohistochemistry. In subcutaneously implanted HCC tumor composed of Huh-7 cells and LX2 cells under the stimulation of cancer cell-derived exosomes, vascular density was significantly higher than that of the control group, where as the inhibitor of the miRNA-21 reversed the tumor volume (Fig. 7a and b). At the same time, we found that CAFs were significantly closer to vessels than other cells in the tumor by immunofluorescence (Additional file 10: Figure S9), indicating that they resided in proximity to the developing tumor vasculature. This would be the ideal position, in which CAFs could promote angiogenesis through deposition of angiogenic factors in the vascular basement membrane. In order to determine whether CAFs expressed factors capable of promoting angiogenesis, we compared the mRNA expression profiles of CAFs activated by cancer cell-derived exosomes and normal HSCs. The expressions of VEGF- $\alpha$, MMP2, MMP9, bFGF and TGF- $\beta$ were all increased from CAFs of human HCC cancer cell lines compared with the normal HSCs (Fig. 7c). In vitro experiment showed that when vascular endothelial cells were co-cultured with HSCs stimulated by HCC cell-derived exosomes, the proliferation of cells was improved (Fig. 7d), and the ability of cells to form tubular structures was significantly enhanced (Fig. 7e). When the miRNA-21 inhibitor and AKT inhibitor were added, the angiogenesis ability of CAFs was remarkably reduced (Fig. 7c and d). The above-mentioned results suggested that HCC cell-derived exosomes could activate CAFs, leading to improved tumor growth through angiogenesis.

\section{MiRNA-21 correlates with prognosis of HCC patients}

To extend current knowledge to HCC patients, we first examined serum exosomes isolated from $\mathrm{HCC}$ patients and healthy controls by electron microscopy and Western blotting analysis (Fig. 8a-d). Consistent with previous findings, a substantially increased excretion of serum exosomes was detected in HCC patients compared with the healthy controls [32]. Moreover, we investigated the expression of exosomal miRNA-21 in different serum samples (10 healthy controls and 85 HCC patients). Figure $8 \mathrm{e}$ shows that the serum expression of exosomal miRNA-21 was increased in HCC patients compared with the healthy controls. To further determine the correlation between miRNA-21 and clinical pathological features, the HCC patients were divided into two groups (low and high) according to the expression scores of miRNA-21 (Fig. 8f). As shown in Fig. 8g, high miRNA-21 expression (score = 2 and 3) could well predict the poor overall survival and poor disease-free survival. In HCC patients, correlation analysis showed that there was a positive correlation between miRNA-21 in serum and miRNA-21 in tissue (Fig. 8h). In addition, high miRNA-21 expression was closely related to high expressions of fibroblast marker $(\alpha-S M A)$, cell proliferation (Ki67 staining) and vessels (Fig. 8i). Taken together, these data showed that miRNA-21 was differentially expressed in liver cancer, and its high expression level in HCC tissues predicted poor outcome.

In summary, our results showed that tumor-derived exosomal miRNA-21 could convert HSCs to CAFs via down-regulating PTEN and activate PDK1/AKT signaling pathway to promote angiogenesis. Our findings also indicated that tumor-derived exosomal miRNA-21 played an important role in intercellular communication for fostering an inflammatory microenvironment and promoting tumor invasion through angiogenesis (Fig. 9).

\section{Discussion}

As a dynamic system orchestrated by intercellular communications, tumor microenvironment is responsible for tumor progression and metastasis. Therefore, it is necessary to study the interaction between tumor and stroma mediated by exosomes. In our study, we first analyzed the different profiles of exosomal miRNAs between HCC cells and normal liver cells. Then, we identified that miRNA-21 was directly transferred from tumor cells to HSCs in tumor parenchyma via exosomes, and miRNA-21 could convert HSCs to CAFs by down-regulating its target PTEN to activate PDK1/AKT signaling pathway. In addition, CAFs promoted tumor development by angiogenesis through secreting IL- 6 and IL- 8 . The crosstalk between tumor cells and HSCs further elucidated the molecular mechanism of HCC invasion, and explained why liver cancer was highly invasive. Furthermore, our data indicated that high miRNA-21 expression in serum exosomes was correlated with low survival rate, holding important implications for efficient prevention and therapeutic strategies. The involvement of miRNAs in cancer shows that the expressions of several miRNAs are dysregulated in neoplastic tissues [33]. The identification of several targets of miRNAs, which are actually classical oncogenes or tumor suppressors, has led to the widely accepted idea that miRNAs play pivotal roles in cancer initiation, progression and metastasization [34]. In another elegant study employing K-ras G12D non-small-cell lung cancer (NSCLC) mouse model, the results show that incidence of lung tumors is significantly high in miRNA-21-overexpressing mice. Consistent with this finding, deletion of miRNA-21 has resulted in suppression of Kras-driven transformation in vitro and tumor development in vivo [35]. Our data demonstrated that tumor-derived exosomal miRNA-21 converted HSCs to CAFs in HCC. Moreover, stimulation of exosomal 
a

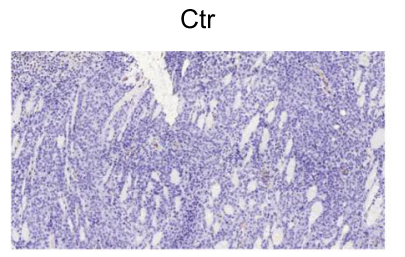

LM3-EXO

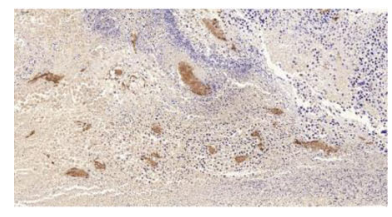

LM3-EXOmiRNA-21-/-

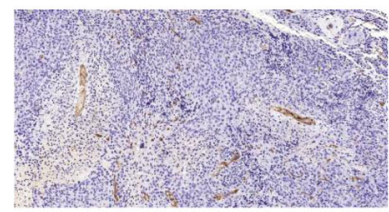

b MVB

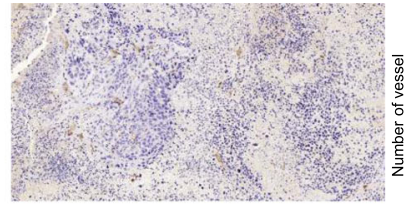

97H-EXO

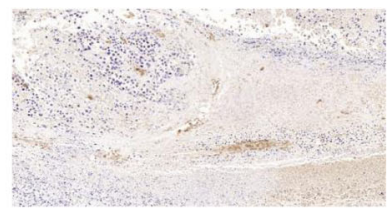

97H-EXOMiRNA-21-/-

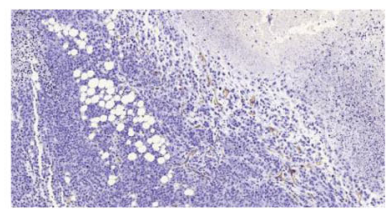

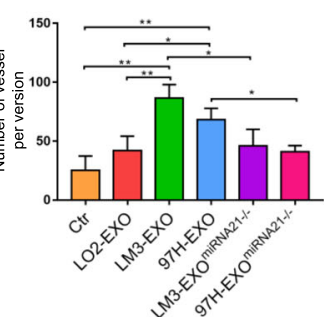

C
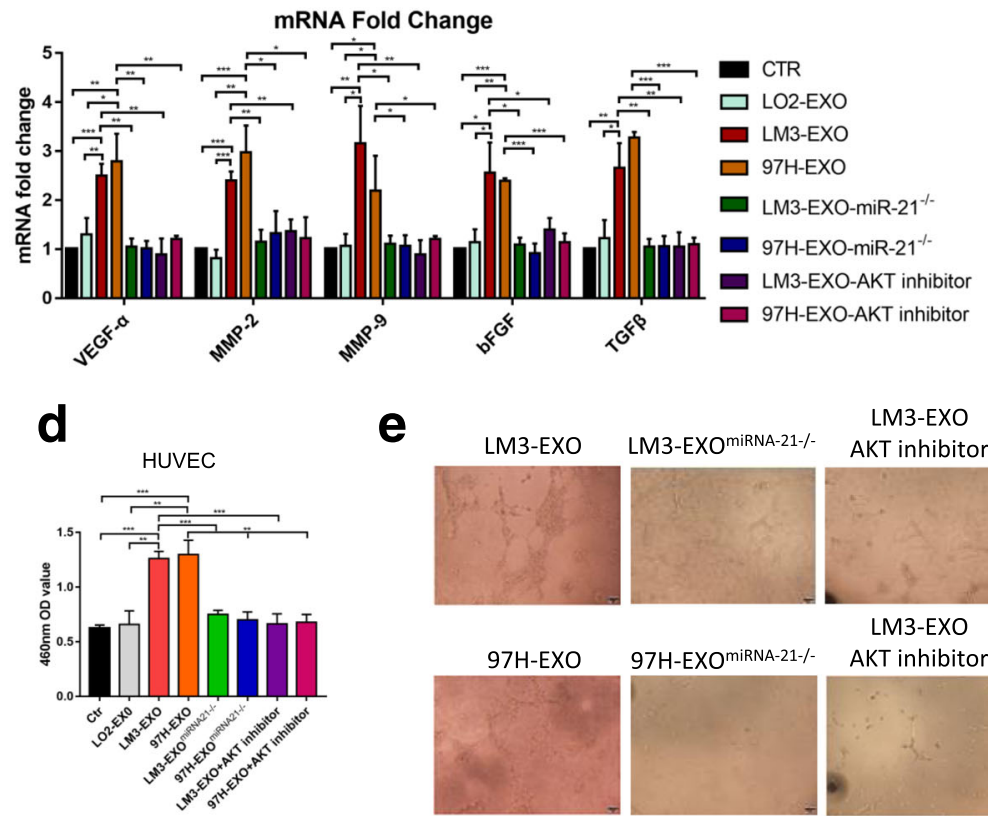

e
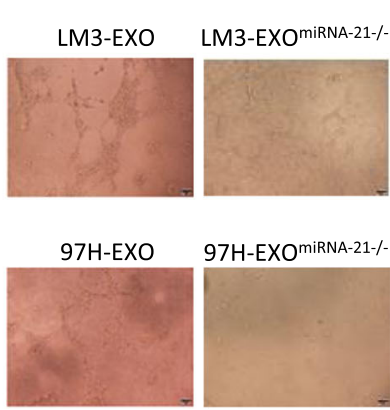

LM3-EXO

AKT inhibitor

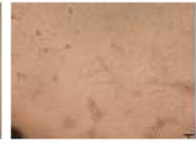

LM3-EXO

97H-EXOMiRNA-21-/

AKT inhibitor

Fig. 7 Activated HSCs promote angiogenesis $\mathbf{a}$, $\mathbf{b}$ Immunohistochemistry staining of CD31 staining in subcutaneously implanted tumor under different conditions. $\mathbf{c}$ qRT-PCR assay indicated gene expression levels of HSCs treated with exosomes derived from different cells in the presence of miRNA-21 inhibitor or AKT inhibitor. Experiments were performed at least in triplicate. $\mathbf{d}$, e The proliferation of HUVECs with exosomes derived from different cells in the presence of miRNA-21 inhibitor or AKT inhibitor was tested by CCK-8 assay and tube formation assay. Data are mean \pm SEM from three independent experiments, ${ }^{*} p<0.05,{ }^{* *} p<0.01,{ }^{* * *} p<0.001$ by unpaired Student's t-test

miRNA-21 from HCC cells had a positive correlation with tumor volume in nude mice. Meanwhile, high miRNA-21 expression in HCC predicted a poor outcome. Previous studies have mainly focused on miR-21 overexpression in cancer cells, which promotes cellular proliferation, evasion of apoptosis, EMT and invasion. However, more and more attention has been paid to the role of miR-21 in CAFs. In a study on colorectal cancer, miRNA-21 expression is increased in stromal cells compared with normal tissues, and the ectopic expression of miR-21 drives the trans-differentiation of fibroblasts into myofibroblasts and increases invasion in vitro [36]. MiRNA-21 expression in lung fibroblasts may trigger fibroblast trans-differentiation into CAFs, supporting cancer progression. Furthermore, patients with miR-21-high CAFs exhibit lower survival 


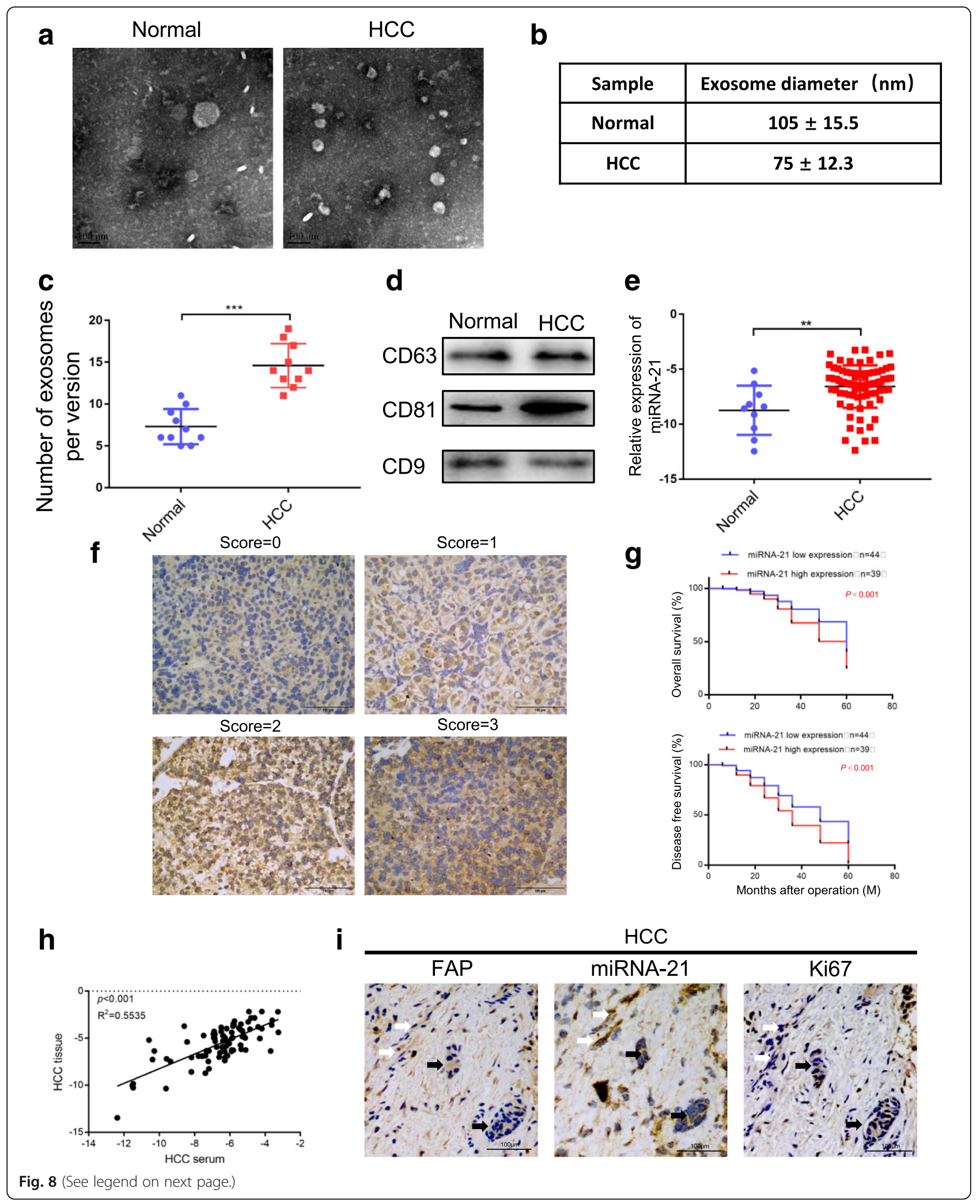


(See figure on previous page.)

Fig. 8 miRNA-21 is associated with HCC progression. a - d Exosomes in normal and HCC serums were detected by electron microscopy. e miRNA-21 expression level in serum exosomes from healthy donors and primary HCC patients. Data are presented as mean \pm s.d. Student's t-test was used to analyze the data. ${ }^{* *} p<0.01$. $\mathbf{f}$ In situ hybridization assay of HCC samples and scores of miRNA-21 level. Representative images were shown. $\mathbf{g}$ Kaplan-Meier plots of overall survival and disease-free survival of 83 HCC patients, stratified by expression of miRNA-21. Survival data were analyzed by the Kaplan-Meier method and log-rank test. $\mathbf{h}$ The correlation analysis between expression of miRNA-21 in serum and expression of miRNA-21 in HCC was detected. $\mathbf{i}$ In situ hybridization of miRNA-21 in combination with IHC staining of HSCs markers (FAP) and proliferation markers (Ki67) on serial sections of human HCC tissues. White arrows indicate CAFs; black arrows indicate vessels

compared with those with miR-21-low CAFs [37]. In summary, CAFs may further secrete exomal-miR21 to promote HCC progression. Future work will be required to study the role of miRNA-21 in liver cancer.

PTEN stands for phosphatase and TENs in homolog deleted on chromosome 10, and it is a classical tumor suppressor gene located in the 10q23 region of chromosome 10 encoding for a 403-amino acid multifunctional protein (predicted MW $47 \mathrm{kDa}$ ), which possesses lipid and protein phosphatase activities [38, 39]. PTEN functions as a classical tumor suppressor, and it is mainly involved in the homeostatic maintenance of the AKT cascade [40]. PI3K, a lipid kinase activated by receptor tyrosine kinases, G protein-coupled receptors and RAS activation, converts the lipid second messenger phosphatidylinositol 4,5-bisphosphate (PIP2) to phosphatidylinositol 3,4,5-trisphosphate (PIP3). PIP3 recruits PDK1 and AKT to the plasma membrane, where AKT is phosphorylated on Thr308 by PDK1. By dephosphorylating PIP3 to PIP2, PTEN reverses the action of PI3K, thereby hampering all downstream functions controlled by the AKT pathway, such as cycle progression, lipid synthesis and stimulation of angiogenesis. We found that decreased PTEN expression disturbed by HCC cell-derived exosomes increased phosphorylation levels of PDK1 and AKT, regulated lipid metabolism and promoted the release of angiogenic substances (VEGF, MMP2, MMP9, bFGF and TGF- $\beta$ ). PTEN also controls cell-cycle progression by decreasing the level of cyclin D1 in the nucleus and regulates cellular senescence. Consistent with previous studies, our results indicated that HSCs with low expression of PTEN under the stimulation of HCC cell-derived exosomes also showed high level of cyclin D1 and increased proportion of cells in the $S$ phase.

Of the factors that were found to be induced by CAFs after co-culture with HCC cell-derived exosomes, the up-regulation of VEGF, MMP2, MMP9, bFGF and TGF- $\beta$ was especially relevant, since these factors are known to promote cancer growth, invasion and angiogenesis through autocrine or paracrine signaling [41-43]. Additionally, these cytokines are associated with advanced stages of breast cancer and a poor clinical outcome. VEGF can also stimulate angiogenesis and it is significantly associated with a poor survival [44]. MMP2 and MMP9 are known multifunctional proteins. Data from diverse experimental models have indicated that these proteases affect cellular activities, including proliferation and survival, gene expression as well as multiple aspects of inflammation. Previous study has reported associations between high expression of MMP2 or MMP9 and tumor aggressiveness in liver cancer [45]. As a highly

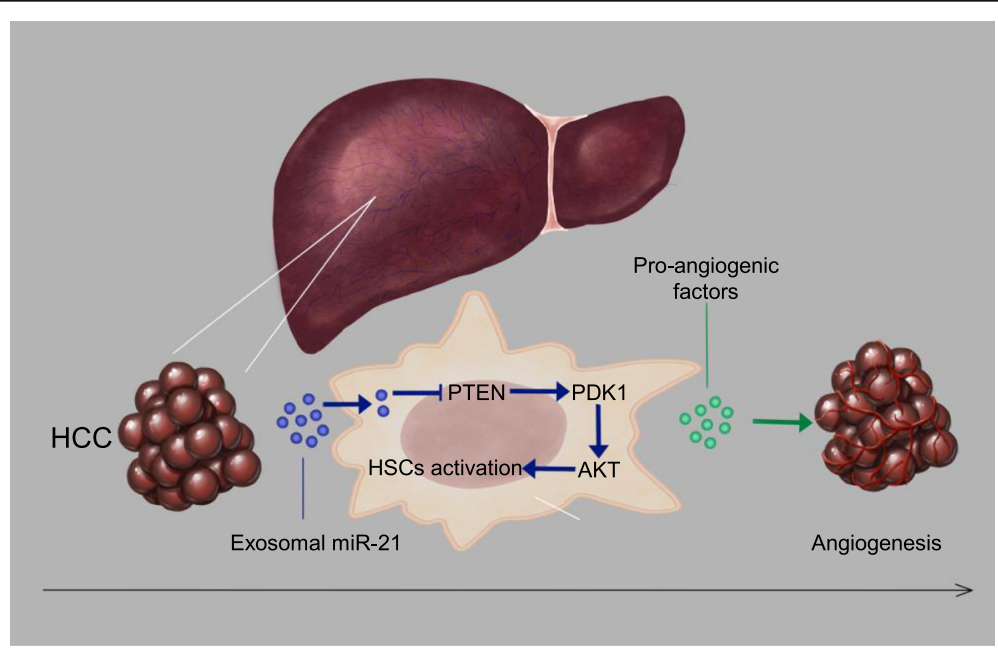

Fig. 9 Proposed schematic diagram of HCC exosomal miR-21-mediating HSCs activation to promote angiogenesis of HCC 
angiogenic molecule, bFGF is of particular interest since it is capable of promoting both the proliferation and migration of endothelial cells in various tumor models, and it can function synergistically with other factors to promote angiogenesis [46]. In summary, we found that HCC cell-activated CAFs could induce the expressions of several factors related to angiogenesis and tumor progression in HCC.

Aberrant activation of lipogenesis is a dominant oncogenic event in human HCC. Importantly, no significant differences were detected in the extent of de novo lipogenesis with regard to HCC etiology, suggesting that exacerbated lipogenesis was a general molecular phenotype in hepatocarcinogenesis. Indeed, previous reports have demonstrated that both hepatitis $\mathrm{B}$ and $\mathrm{C}$ viruses are able to induce FASN expression [47, 48], and over-expression of FASN is a typical feature of liver cancer under another predisposing condition, the alcoholic steatohepatitis [49]. Furthermore, a rat model of insulin-induced hepatocarcinogenesis is characterized by strong up-regulation of FASN [50], which resembles the occurrence of HCC in human affected by type II diabetes mellitus and/or metabolic syndrome, two clinical conditions associated with an increased risk of liver cancer development [51]. Our data suggested that abnormal lipid metabolism was present not only in cancer cells but also in stromal cells. CAFs also expressed a high level of FASN with its maintenance protein USP2a and phosphorylated ACLY. Abnormal lipid accumulation was also coupled with the activation of HSCs.

\section{Conclusions}

Our results indicated that tumor-derived exosomal miRNA-21 could convert HSCs to CAFs by decreasing PTEN, leading to activated PDK1/AKT signaling pathway in HCC. In addition, CAFs exhibited increased secretion of VEGF, MMP2, MMP9, bFGF and TGF- $\beta$, promoting angiogenesis. Meanwhile, we found aberrant activation of lipogenesis in activated HSCs. More importantly, high expression of miRNA-21 in serum exosomes showed a positive correlation with survival in HCC patients. Our study elucidated a new molecular mechanism underlying the crosstalk between tumor cells and HSCs during tumor progression, which greatly contributed to efficient prevention and therapeutic strategies for liver cancer.

\section{Additional files}

Additional file 1: Table S1. Sequences of primers and miRNA-inhibitor/ mimic used in the study. (DOCX $14 \mathrm{~kb}$ )

Additional file 2: Figure S1. The expression of a-SMA in normal liver tissue was negative. (TIF $740 \mathrm{~kb}$ )
Additional file 3: Figure S2. Tumor-derived exosomes activated HSCS in vitro. a Flow cytometry assays of cell cycle showed the increasing $\mathrm{S}$ phase in HCC derived-exosomes treated HSCs. b HSCs exhibited stronger cell contractility during the stimulation of HCC derived-exosomes. C Proinflammatory cytokines of activated HSCs were identified to be upregulated by HCC derived-exosomes treatment. (TIF $1541 \mathrm{~kb}$ )

Additional file 4: Figure S3. Tumor-derived exosomes activated HSCS in vivo. a Immunofluorescence imaging showed the delivery of $97 \mathrm{H}-$ labeled exosomes (green) to FAP-labeled CAFs (red). Yellow arrows represent delivered exosomes. (TIF $192 \mathrm{~kb}$ )

Additional file 5: Figure S4. Tumor-derived exosomes activated HSCS in vivo. a Immunohistochemistry imaging of Ki67 showed the proliferation of HSCs with the stimulation of HCC derived exosomes. Black arrows show proliferated cells, white arrows indicate non-proliferated cells. (TIF $713 \mathrm{~kb}$ )

Additional file 6: Figure S5. Detection of miRNA-21 in HCC cells and HCC cell-derived exosomes treated HSCs. QPCR array demonstrated the high expression of miRNA-21 in HCC cell lines and increased expression of HSCs treated with HCC cell-derived exosomes. (TIF $1120 \mathrm{~kb}$ )

Additional file 7: Figure S6. MiRNA-21 mediates HSC activation. Cell contraction assay (a), Edu staining assay (b) and flow cytometry assay of cell cycle (c) were used to detect the activation of HSCs transfected with miR-21 mimic or negative control (miR-RC). (TIF $1626 \mathrm{~kb}$ )

Additional file 8: Figure S7. Exosomal miRNA-21 activates HSCs via PTEN/PDK1/AKT signaling axis. Immunofluorescence assay of a-SMA (a), Edu staining assay $(b, c)$, flow cytometry assay (d), migration assay (e, f), wound-healing assay (g) of HSCs treated with exosomes derived from different cells co-cultured with miRNA-21 inhibitor or AKT inhibitor. Representative images were shown, and migrated cells were counted. (TIF $1699 \mathrm{~kb}$ )

Additional file 9: Figure S8. Exosomal miRNA-21 activates HSCs via PTEN/PDK1/AKT signaling axis. The HSCs were treated with exosomes derived from different cells co-cultured with miRNA-21 inhibitor or AKT inhibitor. And the cell contraction assay (a), CCK-8 proliferation assay (b) were used to detect the activation of HSCs. c qPCR array demonstrated that the downregulation of proinflammatory cytokines was caused by inhibition of miRNA-21 and AKT activation. (TIF $1736 \mathrm{~kb}$ )

Additional file 10: Figure S9. Activated HSCs promote angiogenesis. a Immunofluorescence imaging showed the activated CAFs (FAP) and the vessels (red). Yellow arrows represent activated CAFs. (TIF $415 \mathrm{~kb}$ )

\section{Abbreviations}

ACLY: ATP citrate lyase; AKT: v-akt murine thymoma viral oncogene homolog; bFGF: Basic fibroblast growth factor; FAP: Fibroblast activation protein; FASN: Fatty acid synthase; FSP: Fibroblast stimulated protein; HCC: Hepatocellular carcinoma; MMP2: Matrix metalloproteinase 2; MMP9: Matrix metalloproteinase 9; PDK1: 3-Phosphoinositide-dependent protein kinase-1; PTEN: Gene of phosphate and tension homology deleted on chromsome ten; TGF- $\beta$ : Tumor Growth Factor- $\beta$; USP2: Ubiquitin-specific protease 2; VEGF-a: Vascular endothelial growth factor-a; $a$-SMA: a-smooth muscle actin

\section{Acknowledgements}

The authors thank all members of the Department of Hepatobiliary Surgery for assistance in various aspects of this work.

\section{Funding}

This work was supported by grants from the National Natural Science Foundation of China (Grant No. 81670566 and No. 81872359), Jiangsu Province's Key Provincial Talents Program (Grant No. ZDRCA2016066).

Availability of data and materials

All data generated or analysed during this study are included in this published article [and its supplementary information files].

\section{Authors' contributions}

XS provided the direction and guidance throughout the preparation of this manuscript. JH gave a lot of guidance to the experimental design and 
participated in the discussion process of the whole experiments. $Y Z$ wrote the whole manuscript. HR and BD made significant revisions to the manuscript. $J$ and LS took part in the experiment. All authors have read and approved the final manuscript.

\section{Ethics approval and consent to participate}

The present study was authorized by the Ethics Committee of Nanjing Drum Tower Hospital. All procedures performed in studies were in accordance with the ethical standards. All patients and volunteers were anonymous and provided written informed consent.

\section{Consent for publication}

Not applicable.

\section{Competing interests}

The authors declare that they have no competing interests.

\section{Publisher's Note}

Springer Nature remains neutral with regard to jurisdictional claims in published maps and institutional affiliations.

Received: 31 July 2018 Accepted: 16 November 2018

Published online: 27 December 2018

\section{References}

1. Singal $A G$, El-Serag HB. Hepatocellular carcinoma from epidemiology to prevention: translating knowledge into practice. Clin Gastroenterol Hepatol. 2015;13:2140-51

2. Bruix J, Gores GJ, Mazzaferro V. Hepatocellular carcinoma: clinical frontiers and perspectives. Gut. 2014;63:844-55.

3. Kubo N, Araki K, Kuwano H, Shirabe K. Cancer-associated fibroblasts in hepatocellular carcinoma. World J Gastroenterol. 2016;22:6841-50.

4. Affo S, Yu LX, Schwabe RF. The role of Cancer-associated fibroblasts and fibrosis in liver Cancer. Annu Rev Pathol. 2017;12:153-86.

5. Lau EY, Lo J, Cheng BY, Ma MK, Lee JM, Ng JK, Chai S, et al. Cancerassociated fibroblasts regulate tumor-initiating cell plasticity in hepatocellular carcinoma through c-met/FRA1/HEY1 signaling. Cell Rep. 2016;15:1175-89.

6. Huang L, Xu AM, Liu S, Liu W, Li TJ. Cancer-associated fibroblasts in digestive tumors. World J Gastroenterol. 2014;20:17804-18.

7. Serini G, Gabbiani G. Mechanisms of myofibroblast activity and phenotypic modulation. Exp Cell Res. 1999:250:273-83.

8. Sharon Y, Raz Y, Cohen N, Ben-Shmuel A, Schwartz H, Geiger T, Erez N. Tumor-derived osteopontin reprograms normal mammary fibroblasts to promote inflammation and tumor growth in breast cancer. Cancer Res. 2015;75:963-73.

9. Dror S, Sander L, Schwartz H, Sheinboim D, Barzilai A, Dishon Y, Apcher S, et al. Melanoma miRNA trafficking controls tumour primary niche formation. Nat Cell Biol. 2016;18:1006-17.

10. Rasanen K, Vaheri A. Activation of fibroblasts in cancer stroma. Exp Cell Res. 2010:316:2713-22.

11. Jung DW, Che ZM, Kim J, Kim K, Kim KY, Williams D, Kim J. Tumor-stromal crosstalk in invasion of oral squamous cell carcinoma: a pivotal role of CCL7. Int J Cancer. 2010;127:332-44.

12. Scherz-Shouval R, Santagata S, Mendillo ML, Sholl LM, Ben-Aharon I, Beck $\mathrm{AH}$, Dias-Santagata $\mathrm{D}$, et al. The reprogramming of tumor stroma by HSF1 is a potent enabler of malignancy. Cell. 2014;158:564-78.

13. Salido-Guadarrama I, Romero-Cordoba S, Peralta-Zaragoza O, HidalgoMiranda A, Rodriguez-Dorantes M. MicroRNAs transported by exosomes in body fluids as mediators of intercellular communication in cancer. Onco Targets Ther. 2014;7:1327-38.

14. Greening DW, Gopal SK, Mathias RA, Liu L, Sheng J, Zhu HJ, Simpson RJ. Emerging roles of exosomes during epithelial-mesenchymal transition and cancer progression. Semin Cell Dev Biol. 2015:40:60-71.

15. Azmi AS, Bao B, Sarkar FH. Exosomes in cancer development, metastasis, and drug resistance: a comprehensive review. Cancer Metastasis Rev. 2013; 32:623-42.

16. Junttila MR, de Sauvage FJ. Influence of tumour micro-environment heterogeneity on therapeutic response. Nature. 2013;501:346-54.
17. Tang $X$, Hou Y, Yang G, Wang X, Tang S, Du YE, Yang L, et al. Stromal miR 200s contribute to breast cancer cell invasion through CAF activation and ECM remodeling. Cell Death Differ. 2016;23:132-45.

18. Mao B, Wang G. MicroRNAs involved with hepatocellular carcinoma (review). Oncol Rep. 2015;34:2811-20.

19. Quinn SR, O'Neill LA. A trio of microRNAs that control toll-like receptor signalling. Int Immunol. 2011;23:421-5.

20. Challagundla KB, Wise PM, Neviani P, Chava H, Murtadha M, Xu T, Kennedy $\mathrm{R}$, et al. Exosome-mediated transfer of microRNAs within the tumor microenvironment and neuroblastoma resistance to chemotherapy. J Natl Cancer Inst. 2015;107. https://www.ncbi.nlm.nih.gov/pmc/articles/ PMC4651042/.

21. Epstein DM. Special delivery: microRNA-200-containing extracellular vesicles provide metastatic message to distal tumor cells. J Clin Invest. 2014;124:5107-8.

22. Lasser C, Eldh M, Lotvall J. Isolation and characterization of RNA-containing exosomes. J Vis Exp. 2012;(59):e3037.

23. Greuter $\mathrm{T}$, Shah VH. Hepatic sinusoids in liver injury, inflammation, and fibrosis: new pathophysiological insights. J Gastroenterol. 2016;51:511-9.

24. Li S, Yao J, Xie M, Liu Y, Zheng M. Exosomal miRNAs in hepatocellular carcinoma development and clinical responses. J Hematol Oncol. 2018;11:54.

25. Adams BD, Kasinski AL, Slack FJ. Aberrant regulation and function of microRNAs in cancer. Curr Biol. 2014;24:R762-76.

26. Meng F, Henson R, Wehbe-Janek H, Ghoshal K, Jacob ST, Patel T. MicroRNA21 regulates expression of the PTEN tumor suppressor gene in human hepatocellular cancer. Gastroenterology. 2007;133:647-58.

27. Moeini A, Cornella H, Villanueva A. Emerging signaling pathways in hepatocellular carcinoma. Liver Cancer. 2012;1:83-93.

28. Menendez JA, Lupu R. Fatty acid synthase and the lipogenic phenotype in cancer pathogenesis. Nat Rev Cancer. 2007;7:763-77.

29. Liao X, Song L, Zhang L, Wang H, Tong Q, Xu J, Yang G, et al. LAMP3 regulates hepatic lipid metabolism through activating PI3K/Akt pathway. Mol Cell Endocrinol. 2018:470:160-7.

30. Graner E, Tang D, Rossi S, Baron A, Migita T, Weinstein L, Lechpammer M, et al. The isopeptidase USP2a regulates the stability of fatty acid synthase in prostate cancer. Cancer Cell. 2004;5:253-61.

31. Luzzi KJ, MacDonald IC, Schmidt EE, Kerkvliet N, Morris VL, Chambers AF, Groom AC. Multistep nature of metastatic inefficiency: dormancy of solitary cells after successful extravasation and limited survival of early micrometastases. Am J Pathol. 1998;153:865-73

32. Melo SA, Sugimoto $H$, O'Connell JT, Kato N, Villanueva A, Vidal A, Qiu L, et al. Cancer exosomes perform cell-independent microRNA biogenesis and promote tumorigenesis. Cancer Cell. 2014;26:707-21.

33. Lu J, Getz G, Miska EA, Alvarez-Saavedra E, Lamb J, Peck D, Sweet-Cordero A, et al. MicroRNA expression profiles classify human cancers. Nature. 2005; 435:834-8.

34. Nana-Sinkam SP, Croce CM. MicroRNA regulation of tumorigenesis, cancer progression and interpatient heterogeneity: towards clinical use. Genome Biol. 2014;15:445.

35. Hatley ME, Patrick DM, Garcia MR, Richardson JA, Bassel-Duby R, van Rooij E, Olson EN. Modulation of K-Ras-dependent lung tumorigenesis by MicroRNA-21. Cancer Cell. 2010;18:282-93.

36. Bullock MD, Pickard KM, Nielsen BS, Sayan AE, Jenei V, Mellone M, Mitter R, et al. Pleiotropic actions of miR-21 highlight the critical role of deregulated stromal microRNAs during colorectal cancer progression. Cell Death Dis. 2013:4:e684.

37. Kunita A, Morita S, Irisa TU, Goto A, Niki T, Takai D, Nakajima J, et al. MicroRNA21 in cancer-associated fibroblasts supports lung adenocarcinoma progression Sci Rep. 2018;8:8838.

38. Li DM, Sun H. TEP1, encoded by a candidate tumor suppressor locus, is a novel protein tyrosine phosphatase regulated by transforming growth factor beta. Cancer Res. 1997;57:2124-9.

39. Steck PA, Pershouse MA, Jasser SA, Yung WK, Lin H, Ligon AH, Langford LA, et al. Identification of a candidate tumour suppressor gene, MMAC1, at chromosome 10q23.3 that is mutated in multiple advanced cancers. Nat Genet. 1997;15:356-62.

40. Milella M, Falcone I, Conciatori F, Cesta Incani U, Del Curatolo A, Inzerilli N, Nuzzo CM, et al. PTEN: multiple functions in human malignant tumors. Front Oncol. 2015:5:24

41. Saharinen P, Eklund L, Pulkki K, Bono P, Alitalo K. VEGF and angiopoietin signaling in tumor angiogenesis and metastasis. Trends Mol Med. 2011;17: 347-62. 
42. Rhee JS, Coussens LM. RECKing MMP function: implications for cancer development. Trends Cell Biol. 2002;12:209-11.

43. Gordon-Weeks AN, Lim SY, Yuzhalin AE, Jones K, Markelc B, Kim KJ, Buzzelli $\mathrm{JN}$, et al. Neutrophils promote hepatic metastasis growth through fibroblast growth factor 2-dependent angiogenesis in mice. Hepatology. 2017;65: 1920-35.

44. Tez M, Tez S. Serum endostatin predicts tumor vascularity in hepatocellular carcinoma. Cancer. 2003;98:1101 author reply -2 .

45. Naim A, Pan Q, Baig MS. Matrix metalloproteinases (MMPs) in liver diseases. J Clin Exp Hepatol. 2017;7:367-72.

46. Cao Y, Cao R, Hedlund EM. R regulation of tumor angiogenesis and metastasis by FGF and PDGF signaling pathways. J Mol Med (Berl). 2008;86:785-9.

47. Na TY, Shin YK, Roh KJ, Kang SA, Hong I, Oh SJ, Seong JK, et al. Liver $X$ receptor mediates hepatitis $B$ virus $X$ protein-induced lipogenesis in hepatitis B virus-associated hepatocellular carcinoma. Hepatology. 2009;49: $1122-31$.

48. Oem JK, Jackel-Cram C, Li YP, Zhou Y, Zhong J, Shimano H, Babiuk LA, et al. Activation of sterol regulatory element-binding protein $1 \mathrm{c}$ and fatty acid synthase transcription by hepatitis C virus non-structural protein 2. J Gen Virol. 2008;89:1225-30.

49. Yin $\mathrm{HQ}$, Kim M, Kim JH, Kong G, Kang KS, Kim HL, Yoon Bl, et al. Differential gene expression and lipid metabolism in fatty liver induced by acute ethanol treatment in mice. Toxicol Appl Pharmacol. 2007;223:225-33.

50. Evert M, Schneider-Stock R, Dombrowski F. Overexpression of fatty acid synthase in chemically and hormonally induced hepatocarcinogenesis of the rat. Lab Investig. 2005:85:99-108.

51. El-Serag HB, Hampel H, Javadi F. The association between diabetes and hepatocellular carcinoma: a systematic review of epidemiologic evidence. Clin Gastroenterol Hepatol. 2006;4:369-80.

Ready to submit your research? Choose BMC and benefit from:

- fast, convenient online submission

- thorough peer review by experienced researchers in your field

- rapid publication on acceptance

- support for research data, including large and complex data types

- gold Open Access which fosters wider collaboration and increased citations

- maximum visibility for your research: over $100 \mathrm{M}$ website views per year

At $\mathrm{BMC}$, research is always in progress.

Learn more biomedcentral.com/submissions 